\title{
Computer simulations and real-time control of ELT AO systems using graphical processing units
}

\author{
Lianqi Wang, Brent Ellerbroek \\ Thirty Meter Telescope Project, 1111 S. Arroyo Pkwy, Suite 200, Pasadena, CA, 91105, USA
}

\begin{abstract}
The adaptive optics (AO) simulations at the Thirty Meter Telescope (TMT) have been carried out using the efficient, $\mathrm{C}$ based multi-threaded adaptive optics simulator (MAOS, http://github.com/lianqiw/maos). By porting time-critical parts of MAOS to graphical processing units (GPU) using NVIDIA CUDA technology, we achieved a 10 fold speed up for each GTX 580 GPU used compared to a modern quad core CPU. Each time step of full scale end to end simulation for the TMT narrow field infrared AO system (NFIRAOS) takes only 0.11 second in a desktop with two GTX 580s. We also demonstrate that the TMT minimum variance reconstructor can be assembled in matrix vector multiply (MVM) format in 8 seconds with 8 GTX 580 GPUs, meeting the TMT requirement for updating the reconstructor. We also benchmarked applying MVM in GPUs and got $1.7 \mathrm{~ms}$ end-to-end latency with 7 GTX 580 GPUs.
\end{abstract}

\section{INTRODUCTION}

The adaptive optics (AO) systems for future ground based extremely large telescopes are so complex that endto-end numerical simulations in the time domain are an essential part of detailed performance analysis. The AO simulations at TMT for the first light AO system NFIRAOS have been carried out using the $\mathrm{C}$ based multithreaded adaptive optics simulator (MAOS, freely available at http://github.com/lianqiw/maos). ${ }^{1}$ It achieves $>20$ fold speed up compared to the previous MATLAB based linear AO simulator (LAOS), thanks to parallelization and efficient code.

General purpose computing with graphics processing units (GPGPU) has been increasingly used in high performance computing and $\mathrm{AO}$ real time controllers (RTC $)^{2,3}$ due to unprecedented computing power and improved programmability. To improve simulation efficiency and study the potential application for the NFIRAOS RTC, we ported performance-critical parts of MAOS, such as wavefront sensing, wavefront reconstruction, performance evaluation, etc. to GPU using NVIDIA CUDA technology. ${ }^{4}$ We chose the consumer card GTX 580 instead of the Tesla GPU that is dedicated to high performance computing due to its lower cost ( $1 / 4$ the price) and comparative single precision floating point operation capability. We therefore implemented the above routines in GPU with single precision floating point numbers, which proves to be adequate in most cases.

We achieved about a 10 fold speed up for each GTX 580 used compared to a modern quad core CPU with hyper-threading enabled. Each time step of a full scale end to end NFIRAOS simulation takes 0.1 second in a desktop with two GTX 580s, which means a minute of real time can be simulated in slightly more than an hour. This has made possible simulation work that was not feasible in the past, such as simulation based point spread function reconstruction. ${ }^{5}$

In the mean time, we studied several numerical solvers for the minimum variance reconstructor $(\mathrm{MVR})^{6,7}$ planned to be used in the NFIRAOS RTC. We found that 30 iterations of the conjugate gradient (CG) tomography algorithm, from six order 60x60 LGS WFS to six turbulence layers (with four layers oversampled) can be accomplished in 25 milliseconds in a single GTX 580. The Fourier domain preconditioned CG algorithm with 3 iterations can be accomplished in 5 milliseconds, which is only 5 times away from the requirement. However, iterative algorithms are hard to parallelize through multiple GPUs due to bandwidth and latency limitation of the PCI-E interface.

The traditional reconstructor used in RTCs for AO systems is a matrix vector multiply, using a matrix computed by pseudo-inverting the deformable mirror (DM) to wavefront sensor (WFS) gradient influence function

Send correspondence to Lianqi Wang: lianqiw@tmt.org

Adaptive Optics Systems III, edited by Brent L. Ellerbroek, Enrico Marchetti, Jean-Pierre Véran, Proc. of SPIE Vol. 8447, $844723 \cdot$ ( ) 2012 SPIE · CCC code: 0277-786/12/\$18 · doi: 10.1117/12.926500 
(referred to as the least square reconstructor, $\mathrm{LSR}^{8-11}$ ). This algorithm is very suitable for RTCs due to straightforward parallelization. However, this MVM is not suitable ${ }^{6,12}$ for modern AO systems with multiple laser guide stars (LGS) and/or DMs due to increasing difficulty in inverting the matrix and/or poorer performance without the use of statistical priors. Minimum variance reconstructors with advanced solvers, such as conjugate gradients $(\mathrm{CG})$, Fourier domain preconditioned $\mathrm{CG},{ }^{13,14}$ etc have therefore been studied to overcome this obstacle. These advanced solves are usually difficult to implement in a real time RTC due to complexity in parallelization. On the other hand, if we could build the matrix used in MVM using the MVR with advanced solvers, we avoid the two problems associated with the traditional MVM method, yet make the RTC straightforward to implement.

Using an innovative approach, we demonstrated that we can build the matrix for MVM for NFIRAOS in $\sim 80$ seconds and/or update it in $\sim 8$ seconds using 8 GTX 580 GPUs hosted in a single server *. It is also possible to apply the MVM using 8 GTX 580 GPUs in real time within the specified TMT latency of $\leq 1 \mathrm{~ms}$.

The rest of this paper is organized as follows. In Section 2 we give a brief introduction to MAOS. In Section 3 we discussed the porting of MAOS to GPU computing. In Section 4 we describe in detail the implementation of the minimum variance reconstructor in GPU. In Section 5 we describe how we build the matrix for MVM in GPU and discuss how it can be applied in real time. Finally Section 6 gives the conclusion.

\section{MULTI-THREADED ADAPTIVE OPTICS SIMULATOR}

The Multi-threaded Adaptive Optics Simulator (MAOS) started as a reimplementation of the algorithms in the original, MATLAB ${ }^{\circledR}$-based linear AO simulator (LAOS). MAOS is written in C language with a function oriented design. MAOS is a complete, end to end AO simulator that is completely configurable through easyto-read configuration files and command line options, and thus suitable for exploring large parameter spaces. MAOS tries its best to check the configuration for any apparent errors or conflicts. The up-to-date version of documentation and MAOS code can be obtained from https://github.com/lianqiw/maos/. MAOS compiles and runs in Linux, Windows (Cygwin), and Mac OS X.

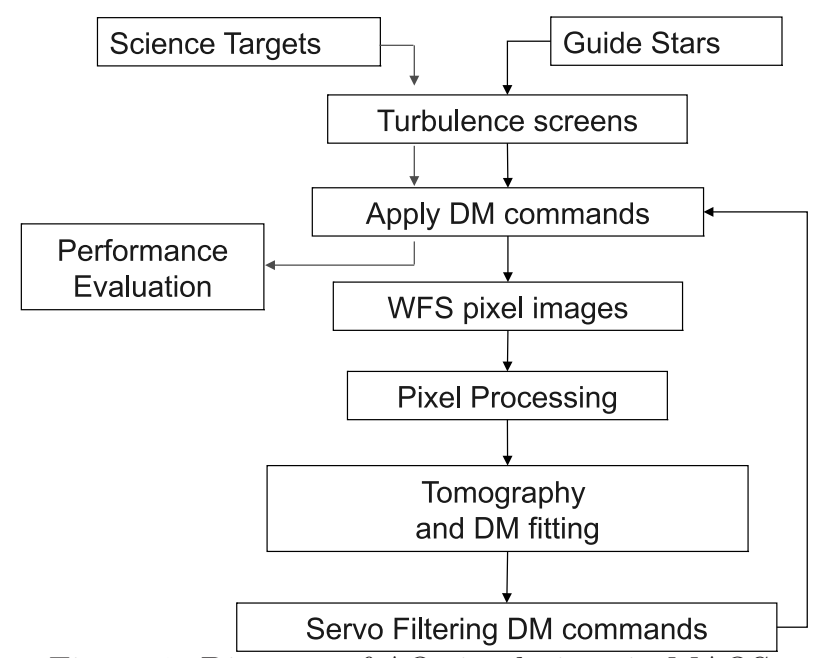

Figure 1: Diagram of AO simulations in MAOS.

Figure 1 shows the block diagram of a basic AO simulations implemented in MAOS. Atmospheric turbulence is represented as one or several discrete optical path difference (OPD) screen(s) at different heights that evolve according to frozen flow with given wind velocity. The resulting aberrations, after corrected by deformable mirror(s) are sensed by one or multiple natural guide star (NGS) or laser guide star (LGS) Shack-Hartmann wavefront sensor(s) (WFS). The sensors can be simulated as idealized wavefront gradient sensors, best Zernike fit tilt sensors, or a physical optics WFS using user specified pixel characteristics and a matched filter ${ }^{15}$ pixel processing algorithm. The laser guide star wavefront sensing model includes the impact of guide star elongation

\footnotetext{
${ }^{*}$ Tyan B7015F72V2R, http://www.tyan.com/product_SKU_spec.aspx?ProductType=BB\&pid=412\&SKU=600000150
} 


\begin{tabular}{|c|c|c|}
\hline Timing (seconds) & CPU (quad-core) & GPU (1 GTX 580) \\
\hline \hline Physical Optics WFS & 1.36 & 0.13 \\
\hline Science RMS WFE & 0.47 & 0.05 \\
\hline Tomography (CG30) & 0.20 & 0.025 \\
\hline Tomography (FDPCG3) & 0.11 & 0.007 \\
\hline DM Fitting (CG4) & 0.03 & 0.0025 \\
\hline Total (Phy. WFS) & 2.0 & 0.20 \\
\hline
\end{tabular}

Table 1: Timing on single GTX $580 \mathrm{GPU}$ with $2.8 \mathrm{GHz}$ Intel Core i7 quad-core CPU versus using the CPU alone. The total is less than the sum of components because these steps are running in parallel for better resource utilization.

for a specified sodium layer profile and (optionally) a polar coordinate CCD. The tomographic wavefront reconstruction then estimates the turbulence at one or several different ranges using the pseudo open loop gradients measured by the WFS, using one of several different computationally efficient implementation of a minimum variance reconstruction algorithm as described in. ${ }^{6}$ These reconstructed turbulence screens are then fit to the actuators on one or several deformable mirrors (DMs) to achieve the best possible correction over a specified field of view (FoV). Performance evaluation is done in terms of RMS wavefront error, Strehl ratio, and/or point spread function (PSFs) at a few science objects in the target FoV, which might be different from the FoV used for the fitting step. A range of additional specified features are implemented, such as telescope wavefront aberrations and the ad hoc and minimum variance "split tomography" control algorithms. ${ }^{16-18}$ MAOS can also be configured to evaluate the performance of tomography alone, DM fitting alone, alias-free WFS, etc. For more detailed description, please see. ${ }^{1}$

\section{PROGRAMMING IN GPU}

We ported mission-critical parts of MAOS, including wavefront sensing, wavefront reconstruction, performance evaluation etc, to run on GPUs using NVIDIA CUDA technology. The selected Nvidia GTX 580 GPU has a total of 512 CUDA cores (or stream processors, equivalent to simplified/dedicated CPU cores) arranged in 16 multiprocessors. It is capable of 1581 GFlops of single precision floating point calculations and 192 GB/s device memory bandwidth. These performance numbers are at least ten times of the state-of-the-art quad core CPUs (i.e., Intel Core i7 2600K). In reality, an average speed up of 10 times is generally observed by porting CPU codes to run on GPUs. Therefore, it is very appealing to run simulations on GPU to improve the simulation speed.

To run codes on GPU with CUDA, we first need to copy data from CPU side memory to GPU memory. Then with "kernel" calls, we can launch many threads that work simultaneously on different parts of the data in user-defined pattern. The finished result can then be copied back to CPU side memory or used for follow up kernels. The "kernel" calls can be scheduled in sequence in streams, and different streams can execute in parallel.

The following sections were ported to GPU computing,

1. Wavefront sensing: including ray tracing from the atmosphere and deformable mirrors to the pupil grid, multiple FFTs per subaperture to get images sampled on detectors, photon and read out noise, gradients calculation from subaperture images, etc.

2. Performance evaluation: including RMS wavefront error computation and point spread function calculation.

3. The wavefront reconstruction: including tomography and deformable mirror fitting for the minimum variance reconstructor.

Significant improvements in speed have been achieved, without losing accuracy due to using single precision floating point numbers. Table 1 shows the timing comparison breakdown of a time step of a TMT NFIRAOS end-to-end simulation. With two GTX 580 GPU and a quad core cpu, the timing per AO time step is reduced further to 0.11 seconds.

Although the performance improvement of $\geq 10$ times is significant, we are still far away from the theoretical limits of computing or memory throughput. The main limiting factors are

Proc. of SPIE Vol. $8447844723-3$ 
1. Device memory latency: Each memory operation requires 600 cycles or about $0.3 \mu s$.

2. Kernel launch overhead: Each task is accomplished with a kernel launch which takes $2.3 \mu s$ for asynchronous launch and $6.5 \mu \mathrm{s}$ for each synchronization.

3. GPU to CPU interface bandwidth and latency: The current PCI-E $\times 16$ interface connecting the graphics card with the mother board has only $8 \mathrm{~GB} / \mathrm{s}$ throughput and $10 \mu s$ latency. This single factor makes it almost impossible to use multiple GPUs for tomography using the conjugate gradient algorithm because the synchronization between GPUs slows down the whole process.

\section{WAVEFRONT RECONSTRUCTION IN GPU}

In this section, we will discuss the implementation of the minimum variance wavefront reconstructor in GPU. The algorithm is based on the formulation in. ${ }^{6}$ The wavefront reconstruction can be broken down to two steps, the wavefront tomography and DM fitting.

The tomography step can be summarized by the following equation

$$
\begin{aligned}
\hat{x} & =\left(\tilde{H}_{x}^{T} G_{p}^{T} C_{n}^{-1} G_{p} \tilde{H}_{x}+C_{x}^{-1}\right)^{-1} \tilde{H}_{x}^{T} G_{p}^{T} C_{n}^{-1} g_{o l} \\
& \equiv R_{L}^{-1} R_{R} g_{o l} .
\end{aligned}
$$

Here the $\hat{g}_{o l}$ and $C_{n}$ are the pseudo open loop WFS gradient and its measurement noise covariance, $\tilde{H}_{x}$ is the ray tracing operator for guide star beam from tomography grid (defined on a few layers and sampled at $1 \times$ or $\frac{1}{2} \times$ of the subaperture spacing, as shown in Figure 2) to pupil grid and $G_{p}$ is the influence function from pupil grid to WFS gradients, $C_{x}$ is the covariance of the atmospheric turbulence, and $\hat{x}$ is the reconstructed wavefront defined on the tomography grid. The right hand side operation $G_{x}^{T} C_{n}^{-1}$ is collectively called $R_{R}$ and the left hand side operation $G_{x}^{T} C_{n}^{-1} G_{x}+C_{x}^{-1}$ is collectively called $R_{L}$.

The DM fitting step can be summarized by the following equation

$$
\begin{aligned}
a & =\left(H_{a}^{T} W H_{a}\right)^{-1} H_{a}^{T} W H_{x} \hat{x} \\
& =F_{L}^{-1} F_{R} \hat{x} .
\end{aligned}
$$

Here, $H_{x}$ and $H_{a}$ are ray tracing operators from the tomography grid and DM actuator grid (see Figure 2) to the science focal plane grid along multiple science directions with the weighting defined with $W$. Again the right and left hand side operations are collectively called $F_{R}$ and $F_{L}$.

These tomography and DM fitting operations are relatively straightforward to implement in GPU due to their intrinsic parallelization. The gradient influence function $G_{p}$ can be parallelized over subapertures. The non-standard pixel weighting in partially illuminated subapertures need to be passed to GPU for this calculation. The inverse of tomography covariance matrix $C_{x}^{-1}$ uses the bi-harmonic approximation with periodic boundary conditions so no values need to be passed to GPU. The ray tracing operators $\tilde{H}_{x}, H_{x}, H_{a}$ are just interpolations that can easily be parallelized although one need to be careful when the grids have different sampling for maximum efficiency. The transpose ray tracing operators $\tilde{H}_{x}^{T}$, and $H_{a}^{T}$ need to be implemented as the reverse operation of forward interpolation with atomic operator (fetching memory, accumulating, and storing back done without interruption by other threads) to avoid race condition. The pupil plane weighting matrix $W$ uses gray pixel maps to properly define the pupil, therefore pixel values need to be passed to GPU.

Table 2 shows our measured timing of the operations in tomography. We can see that the achieved GFlops and memory bandwidth are quite below the theoretical throughput, indicating that 1) we are limited by memory throughput, not by Flops, and 2) irregular memory access is severely penalized by device memory operation latency. The inverse ray tracing operation $H_{x}^{T}$ is significantly slower than the forward ray tracing operation $H_{x}$ due to atomic operations used to avoid race condition. 


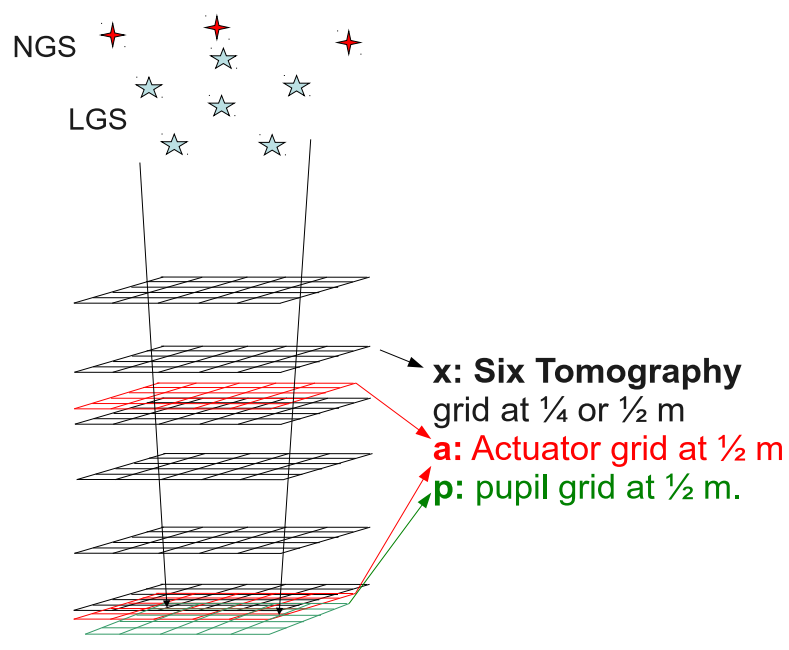

Figure 2: Grid for tomography and DM fitting

\begin{tabular}{|c|c|c|c|c|c|}
\hline & $\mu s$ & $\begin{array}{c}\text { Floating point } \\
\text { operations }(\mathrm{M} \\
\text { Flop })\end{array}$ & $\begin{array}{c}\text { Throughput } \\
\text { (GFlops) }\end{array}$ & $\begin{array}{c}\text { Memory Access } \\
(\mathrm{MB})\end{array}$ & $\begin{array}{c}\text { Bandwidth } \\
(\mathrm{GB} / \mathrm{s})\end{array}$ \\
\hline \hline$H_{x}$ & 161 & 4.6 & 28.4 & 13.7 & 83.2 \\
\hline$H_{x}^{T}$ & 246 & 4.6 & 18.6 & 13.7 & 54.5 \\
\hline$G_{p}$ & 48 & 0.56 & 11.6 & 1.9 & 39.0 \\
\hline$C_{n}^{-1} G_{p}^{T}$ & 91 & 0.68 & 22.6 & 2.1 & 22.6 \\
\hline$C_{x}^{-1}$ & 85 & 1.7 & 19.7 & 5.5 & 63.2 \\
\hline$R_{R}$ & 482 & 5.2 & 32.1 & 16 & 32.1 \\
\hline$R_{L}$ & 603 & 12 & 20.0 & 37 & 59.9 \\
\hline CG30 & 23500 & 382 & 16.2 & 1184 & 49.2 \\
\hline RTC & 1000 & 382 & 382.0 & 1184 & 1184 \\
\hline
\end{tabular}

Table 2: Timing of tomography on a single GTX 580 GPU. The theoretical throughput is 1581 GFlops and 192 GB/s. The row CG30 refers to all the operations for 30 iterations of CG. The row RTC refers to the requirement for RTC with 1 ms latency. 


\section{MVM IN GPU}

As we discussed above, the limited bandwidth $(\sim 10 \mathrm{~GB} / \mathrm{s})$ and latency $(10 \mu s)$ between GPU to GPU/CPU communication interface makes it almost impossible to use multiple GPUs for tomography using the iterative algorithms. Therefore we researched another way to overcome this shortcoming and make an RTC in GPU more attractive. We come back to the easy-to-parallelize matrix vector multiply method. Instead of obtaining the control matrix using the least square reconstructor and/or expensive direct methods such as singular value decomposition (SVD), we can use iterative algorithms, such as CG or FDPCG as described above for the minimum variance reconstructor, to obtain the control matrix in a more acceptable time with improved performance.

\subsection{Obtain the control matrix}

We could obtain the control matrix by solving the identity matrix

$$
\begin{aligned}
E & =F_{L}^{-1} F_{R} R_{L}^{-1} R_{R} I \\
& \rightarrow(7083 \times 7083)^{-1} \times(7083 \times 62311) \times(62311 \times 62311)^{-1} \times(62311 \times 30984) \times(30984 \times 30984) .
\end{aligned}
$$

Here the dimension of each matrix is shown in each parenthesis for the TMT NFIRAOS system. The exponent of -1 indicates that an iterative algorithm instead of a direct inverse of the matrix might be used. We have $n_{a}=7083$ (the number of active DM actuators), $n_{x}=62311$ (number of points in the tomography grid), and $n_{g}=30984$ (the number of LGS WFS gradients. Here split tomography is used where the NGS WFS gradients are used separately to control low order modes only $\left.{ }^{16}\right)$.

Notice that, $R_{R}$ is a matrix with 30984 columns, meaning there are as many sets as tomography plus DM fitting operations to carry out. However, by solving for the transpose of $E$ instead, we can greatly reduce the number of tomography operations,

$$
\begin{aligned}
E^{T} & =R_{R}^{T} R_{L}^{-1} F_{R}^{T} F_{L}^{-1} I \\
& \rightarrow(30984 \times 62311) \times(62311 \times 62311)^{-1} \times(62311 \times 7083) \times(7083 \times 7083)^{-1} \times(7083 \times 7083) .
\end{aligned}
$$

Here we have used the symmetry property of $F_{L}$ and $R_{L}$. In this way, we only need to solve $n_{a}=7083$ sets of tomography plus DM fitting operations, which is more than a factor of 4 reduction. Notice that the operations in $F_{R}$ and $R_{R}$ are mostly ray tracing and wavefront gradient operations, so the transpose operation is similar to the forward operations. Further notice that the DM fitting parameters do not change when the telescope pupil rotates in NFIRAOS or seeing changes. We can solve it using the Cholesky Back Substitution (CBS) method (an exact solution) and reuse it every time we need to initiate or update the control matrix.

To obtain the control matrix the first time, we need to start from a zero guess, so the number of CG or FDPCG iterations needs to be about 10-20 times larger than that required in closed loop simulations that use warm restart. ${ }^{19}$ To update the control matrix when conditions vary (such as pupil rotation or seeing changes), we can simply update from the prior values using warm restart CG or FDPCG, which greatly reduces the number of iterations required.

With precomputed DM fitting results $\left(F_{L}^{-1} I\right)$ calculated in CPU using CBS, it takes about 600 seconds to do the remaining operations $R_{R}^{T} R_{L}^{-1} F_{T}^{T}$ (see Equation 7 ) in a single GTX 580 with 50 iterations of FDPCG in tomography. Notice that the $n_{a}$ columns of $F_{L}^{-1} I$ are strictly independent and can be solved in parallel in different GPUs. So it will only take about 75 seconds with 8 GPUs. In order to update the control matrix when conditions vary, we will only need about 5 FDPCG iterations. Therefore it will take about 8 seconds to update the matrix, which meets the TMT requirement for real time reconstructor updates. Without much modification, this same formulation can be used with other variants of MVR, such as the Frim method. ${ }^{20}$

Figure 3 plots shows the performance obtained using MVM with control matrix computed as described above with FDPCG. We can see that MVM constructed from 50 iterations of FDPCG is about $28 \mathrm{~nm}$ in quadrature worse than the baseline CG30 algorithm. With more FDPCG iterations, the performance of MVM should improve further. 


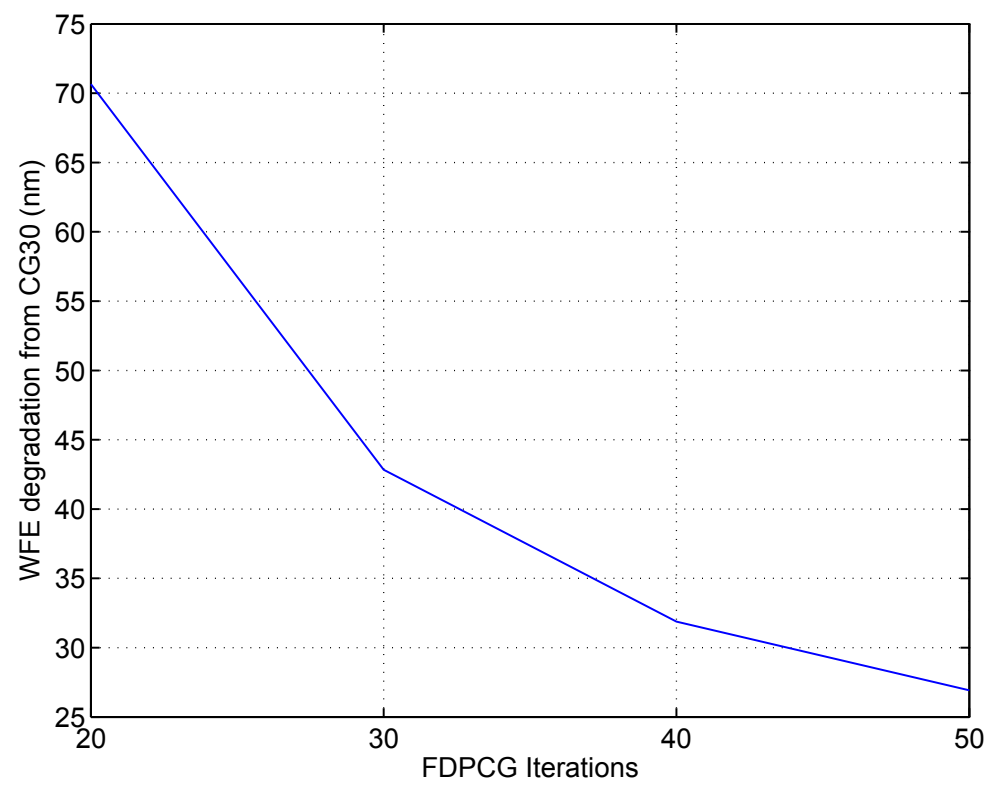

Figure 3: The plot shows the performance using the MVM with a control matrix computed as described above using FDPCG. The $x$ axis shows the number of FDPCG iterations in $R_{L}^{-1}$, and the $y$ axis shows the quadrature difference of the RMS wavefront error with respect to the TMT baseline (30 iterations of warm-restart CG).

\subsection{Apply MVM in GPU based RTC}

Once we have the control matrix ready, it is possible to apply it to gradients to compute actuator commands in GPUs with acceptable latency. ${ }^{2}$ Suppose each element takes 4 bytes, the storage of the matrix will be $4 \times 7083 \times 30984=837$ Mbytes. This fits in the device memory of a single GTX 580 graphics card. But the memory throughput will determine how many GPUs are actually required.

To do the matrix vector multiply, the matrix needs to be brought into GPU from the device memory, and one multiply and accumulation operation (2 floating point operations, or Flops) per matrix element needs to be done. At $1 \mathrm{~ms}$, this requires about 409 giga Flop/s (GFlops) of computing power and 817 giga byte/second (GB/s) of memory throughput. The computing power is within the capacity of a single GTX 580 (1581 GLOPS), but the memory throughput exceeds its capacity of $192 \mathrm{~GB} / \mathrm{s}$. With 8 GPUs, the memory throughput requirement of each GPU decreases to about $102 \mathrm{~GB} / \mathrm{s}$ and should be feasible considering the benign pattern of the memory access.

The next question is whether we can get the gradients into, and actuator commands out of, the GPUs in time. We will partition the problem so that each GPU handle $n_{g} / 8$ number of gradients and accumulate all the actuator command contributions computed from them. The detector read out and gradient computing process is required to complete within 0.5 millisecond after the exposure is done, which is the equivalent of $\sim 620$ gradients every 10 microseconds (roughly the total PCI-E interface latency per transfer). We will distributed these gradients evenly to all the GPUs. This is by far below the PCI-E interface bandwidth $(8 \mathrm{~GB} / \mathrm{s})$ and the latency should not be a problem as long as each transaction of $\sim 620$ gradients completes within 10 microseconds. Finally the actuator commands accumulated in each GPU should then be copied to the CPU memory (level 2 cache is enough to contain all the data) and summed by the CPU. The memory copy from GPU to CPU can be done in about 13 micro-seconds, and the CPU should be able to sum the commands in about 12 micro-seconds.

Based on considerations above, it should be possible to apply the MVM in 8 GTX 580 GPUs in less than 1 ms. By the time TMT RTC gets built, more powerful GPUs should be out, and the latency requirement might be met by fewer cards. 


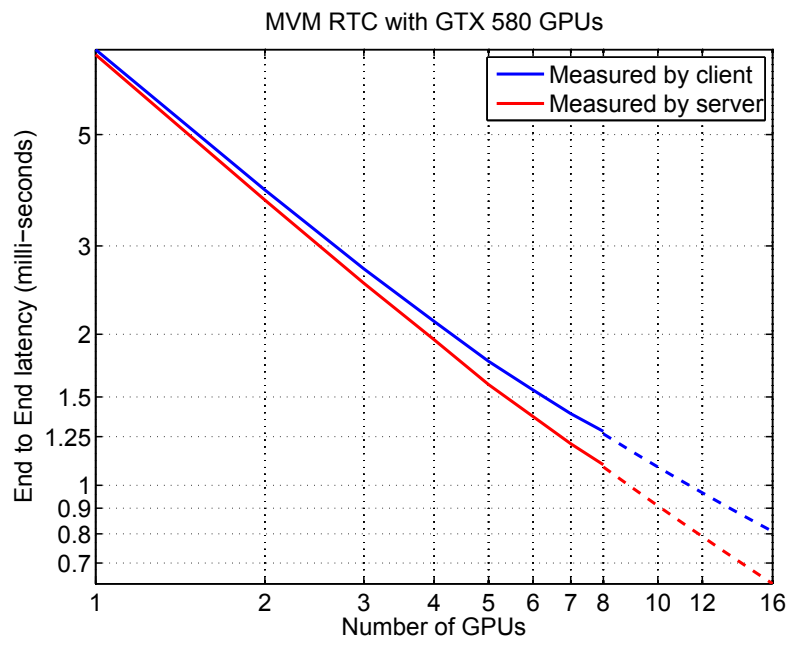

(a) Sending all gradients at once.

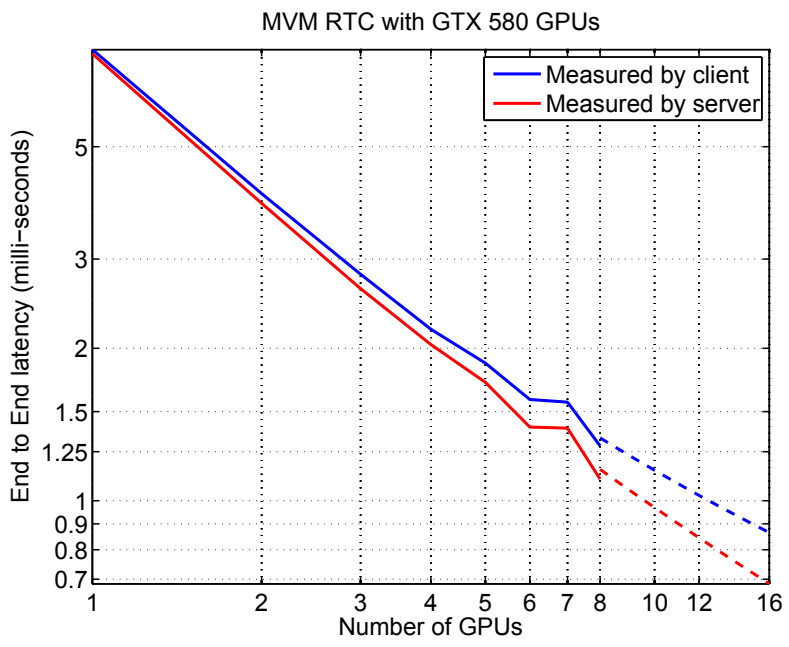

(b) Sending 1300 gradients at each time.

Figure 4: Measured end-to-end latency when server/client are on the same machine

\subsection{Benchmarking MVM in GPU}

We tested applying the MVM in GPUs in a server/client architecture. The server is a standalone 8 GPU server (Occupies $4 \mathrm{U}$ rack space consums maximum of $2 \mathrm{KW}$ ) dedicated to compute the MVM. The client (represent other parts of the RTC) first sends the control matrix to the GPU server for initialization. Both machines run Linux. At every AO time step, the client sends WFS gradients to the server, and the server computes the MVM and send DM commands back to the client. We partition the control matrix column wise so that each GPU work on an equal part of the gradients and the DM commands accumulated by all GPUs are transported to CPU side and summed by CPUs in the end. The end-to-end latency is defined as the time from the beginning of sending gradients to the end of receiving DM commands. We measure this latency in both the client and server. The client always measures longer than the server, due to the TCP/IP communication latency.

\subsubsection{Server/Client on the Same Machine}

The first scenario we benchmarked is for the server/client hosted on the same machine. The server process uses 8 GPUs and 8 CPU cores while the client uses the remaining 4 CPU cores so there is no contention for resource. The server/client still talks through TCP/IP stack, so there is still latency between the communication although network bandwidth limitation is avoided. Figure 5 shows the measured (dashed lines meaning projected) latency. The left panel shows the timing when the client sends the gradients to the server all at once. In this case, the gradient sending and MVM does not overlap. The right panel shows the timing when the client sends successive groups of 1300 gradient to the server. In this case, the latency of successive sending is hidden by the MVM. We don't see a lot of difference here because there is no bandwidth limitation as the server/client is in the same machine and the overhead of executing multiple smaller MVMs (for a subset of the gradients) is canceling the improvement.

\subsubsection{Server/Client connected with 10 Gbit Ethernet}

We then tested another scenario where the server and client run in different machines that are connected by 10 Gbit Ethernet ${ }^{\dagger}$. The left panel in Figure 5 shows the results. The latency is above $0.4 \mathrm{~ms}$ higher with the additional latency caused by Ethernet bandwidth limitation. For unknown reason, we are not quite achieving $1 \mathrm{~GB} / \mathrm{s}$ with $10 \mathrm{Gbit}$ Ethernet. The right panel shows the scattering of the latency when 7 GPU are used (we take out 1 GPU card and replace it with the 10 Gbit Ethernet card). The RMS jitter in the client and server is about 40 and 20 micro-second respectively. The client side has more jitter primarily because of additional latency caused by TCP/IP link.

\footnotetext{
${ }^{\dagger}$ Emulex OCe11102-NX PCIe card
} 


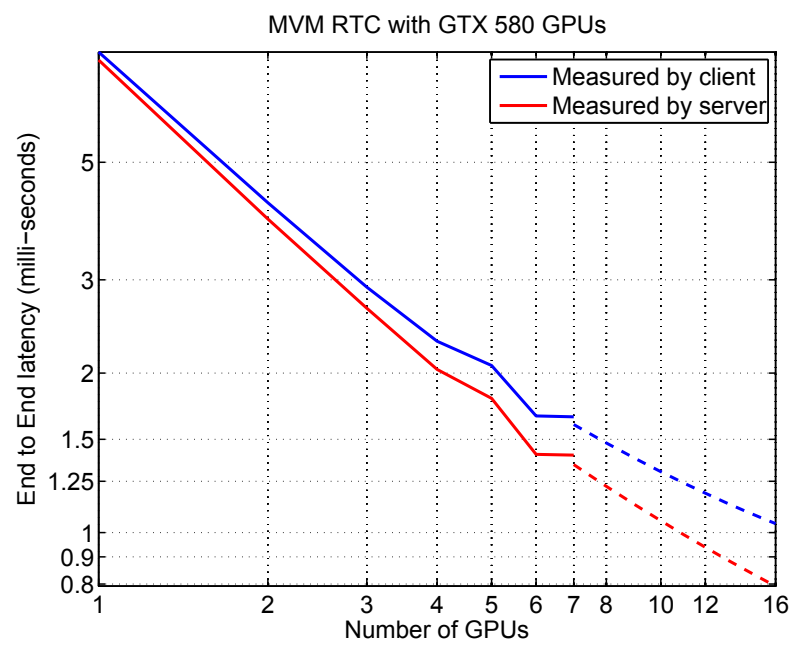

(a) Median latency as a function of number of GPUs used

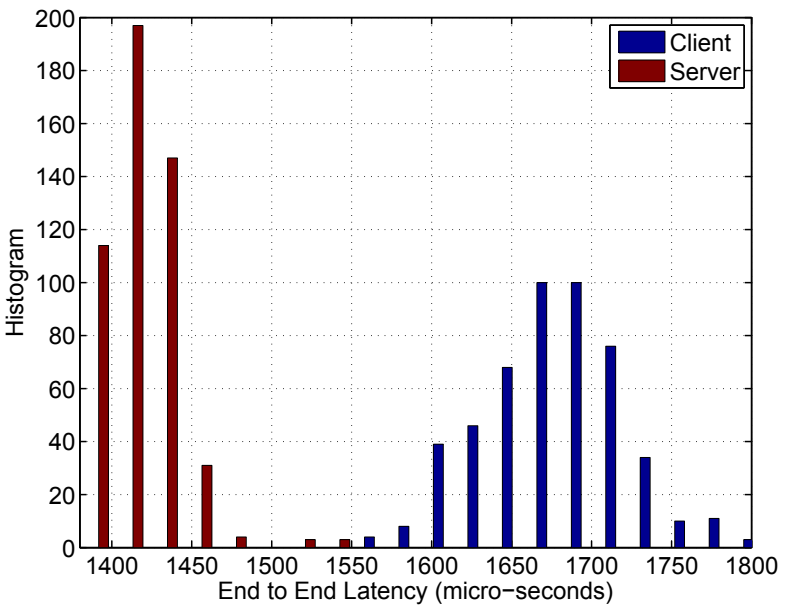

(b) Scattering of latency when 7 GPUs are used

Figure 5: Measured end-to-end latency when server/client run in different machines that are connected by 10 Gbit Ethernet. The client sends successive groups of 860 gradients to the server.

For the $800 \mathrm{~Hz}$ frame rate, a latency of less than $1.25 \mathrm{~ms}$ is required for the RTC to keep up with the loop. When we overlap WFS read out and gradient computation with MVM, we can allow about 1.25 ms latency of MVM. With 7 GPUs, we achieved about $1.7 \mathrm{~ms}$. The latency does not scale linearly with number of GPUs used in a single machine due to additional latency caused by resource contention. However, we think the latency can be reduced to about half with another same 7 GPU server because of bandwidth multiplexing and no resource contention between the servers. With near future hardware, such as the Tesla K20 based on Kepler GPUs, the MVM can probably be done with even less GPUs. The jitter can be reduced by applying real-time patches to the Linux kernel and remove unnecessary tasks in the machines.

\subsection{Other considerations}

\subsubsection{Pseudo open loop gradients}

For the minimum various reconstructor, the gradient vector on the right hand side consists of pseudo open loop (PSOL) gradients, so the final result needs to be differed with the actuator command that was used to form the PSOL gradients:

$$
a=E\left(g_{c l}+G_{a} a_{0}\right)-a_{0} .
$$

Here $a_{0}$ is the DM command vector during WFS integration, and $G_{a}$ is the DM to WFS gradient operator (sparse matrix of dimension $n_{g} \times n_{a}$ ). This formula can be reformatted as

$$
a=E g_{c l}+\left(E G_{a}-I\right) a_{0}
$$

where the first part is the same as the ordinary closed loop matrix vector multiply, while the second part is a correction term using the a prior information. It can be computed quickly with another MVM (the matrix is only $n_{a} \times n_{a}$ ) before the gradients are available and does not contribute to overall latency.

\subsubsection{Minimum variance split tomography}

In the minimum variance split tomography formulation, the NGS gradients need to be adjusted by the LGS tomography results

$$
\hat{a}_{N}=R_{N}\left(g_{N}-G_{N} \hat{x}_{L}\right)
$$

where $g_{N}$ is NGS pseudo open loop gradient and $G_{N}$ is NGS atmosphere to WFS gradient operator. The vector $\hat{x}_{L}$ is the tomography output of Equation 1 . We could precompute the term $R_{N}^{L}=R_{N} G_{N} R_{L}^{-1} R_{R}$ and apply the reconstruction with

$$
\hat{a}_{N}=R_{N} g_{N}-R_{N}^{L} g_{L}
$$

where $R_{N}^{L}$ is only of $n_{g_{N}} \times n_{g_{L}}$ with $n_{g_{N}} \leq 12$. 


\section{CONCLUSION}

In this paper we show that by running performance critical routines in GPUs, we can speed up the adaptive optics simulations by an order of magnitude with consumer graphics cards. Faster simulations makes possible certain studies that require extended time simulation of a real system.

It is also attractive to run the real time controller with GPUs. We demonstrated that we can assemble an end-to-end control matrix from a minimum variance algorithm to be used in a matrix vector multiply manner that is straightforward to implement in RTC. We benchmarked applying MVM with 7 or 8 GPUs and get 1.7 ms end-to-end latency, which can be improved with more GPUs or with future more powerful GPUs.

\section{Acknowledgment}

The TMT Project gratefully acknowledges the support of the TMT collaborating institutions. They are the Association of Canadian Universities for Research in Astronomy (ACURA), the California Institute of Technology, the University of California, the National Astronomical Observatory of Japan, the National Astronomical Observatories of China and their consortium partners, and the Department of Science and Technology of India and their supported institutes. This work was supported as well by the Gordon and Betty Moore Foundation, the Canada Foundation for Innovation, the Ontario Ministry of Research and Innovation, the National Research Council of Canada, the Natural Sciences and Engineering Research Council of Canada, the British Columbia Knowledge Development Fund, the Association of Universities for Research in Astronomy (AURA) and the U.S. National Science Foundation.

\section{REFERENCES}

[1] Wang, L. and Ellerbroek, B., "Fast end-to-end multi-conjugate ao simulations using graphical processing units and the maos simulation code," in [Adaptative Optics for Extremely Large Telescopes II], (2011).

[2] Bouchez, A. H., Dekany, R. G., Roberts, J. E., Angione, J. R., Baranec, C., Bui, K., Burruss, R. S., Croner, E. E., Guiwits, S. R., Hale, D. D. S., Henning, J. R., Palmer, D., Shelton, J. C., Troy, M., Truong, T. N., Wallace, J. K., and Zolkower, J., "Status of the PALM-3000 high-order adaptive optics system," in [Society of Photo-Optical Instrumentation Engineers (SPIE) Conference Series], 7736 (July 2010).

[3] Basden, A., Geng, D., Myers, R., and Younger, E., "Durham adaptive optics real-time controller," Applied Optics 49(32), 6354-6363 (2010).

[4] CUDA, "http://developer.nvidia.com/what-cuda."

[5] Gilles, L., Wang, L., Ellerbroek, B., Correia, C., and Veran, J.-P., "Point spread function reconstruction for laser guide star tomographyadaptive optics," in [Adaptive optics for extrame large telescopes II], (2011).

[6] Ellerbroek, B. L., "Efficient computation of minimum-variance wave-front reconstructors with sparse matrix techniques," J. Opt. Soc. Am. A 19(9), 1803-1816 (2002).

[7] TMT, "NFIRAOS RTC Algorithm," Available at http://www.tmt.org/business/rtc/ .

[8] Fried, D. L., "Least-square fitting a wave-front distortion estimate to an array of phase-difference measurements," J. Opt. Soc. Am. 67, 370-375 (Mar 1977).

[9] Hudgin, R. H., "Wave-front reconstruction for compensated imaging," Journal of the Optical Society of America (1917-1983) 67, 375-378 (Mar. 1977).

[10] Herrmann, J., "Least-squares wave front errors of minimum norm," Journal of the Optical Society of America (1917-1983) 70, 28 (Jan. 1980).

[11] Wallner, E. P., "Optimal wave-front correction using slope measurements," J. Opt. Soc. Am. 73, 1771-1776 (Dec 1983).

[12] Flicker, R., Rigaut, F. J., and Ellerbroek, B. L., "Comparison of multiconjugate adaptive optics configurations and control algorithms for the Gemini South 8-m telescope," in [Society of Photo-Optical Instrumentation Engineers (SPIE) Conference Series], Wizinowich, P. L., ed., 4007, 1032-1043 (July 2000).

[13] Vogel, C. R. and Yang, Q., "Fast optimal wavefront reconstruction for multi-conjugate adaptive optics using the Fourier domain preconditioned conjugate gradient algorithm," Optics Express 14, 7487 (2006).

[14] Yang, Q., Vogel, C. R., and Ellerbroek, B. L., "Fourier domain preconditioned conjugate gradient algorithm for atmospheric tomography," Appl. Opt. 45, 5281-5293 (July 2006). 
[15] Gilles, L. and Ellerbroek, B., "Shack-hartmann wavefront sensing with elongated sodium laser beacons: centroiding versus matched filtering," Appl. Opt. 45(25), 6568-6576 (2006).

[16] Gilles, L. and Ellerbroek, B. L., "Split atmospheric tomography using laser and natural guide stars," Journal of the Optical Society of America A 25, 2427- (Sept. 2008).

[17] Wang, L., Gilles, L., and Ellerbroek, B., "Analysis of the improvement in sky coverage for multiconjugate adaptive optics systems obtained using minimum variance split tomography," Appl. Opt. 50, 3000-3010 (Jun 2011).

[18] Gilles, L., Wang, L., and Ellerbroek, B., "Minimum variance split tomography for laser guide star adaptive optics," European Journal of Control, Special Issue on Adaptive Optics 17, Nb. 3 (2011).

[19] Lessard, L., West, M., Macmynowski, D., and Lall, S., "Warm-started wavefront reconstruction for adaptive optics," Journal of the Optical Society of America A 25, 1147 (Apr. 2008).

[20] Béchet, C., Tallon, M., and Thiébaut, E., "FRIM: minimum-variance reconstructor with a fractal iterative method," in [Society of Photo-Optical Instrumentation Engineers (SPIE) Conference Series], 6272 (July 2006). 